\title{
Ultra-Low Power Pressure Sensing: A Capacitive Based MEMS Approach
}

\author{
Clifford, Mark \\ Kavlico Corporation \\ 14501 Princeton Avenue \\ Moorpark, California 93021
}

\begin{abstract}
:
In a world where wireless sensing and energy harvesting are becoming pervasive technologies, power consumption is becoming more critical for sensor design. This talk will address methods to achieve an ultra-low power consumption sensor using a capacitive sensing element and capacitive interfacing. Using Kavlico's sCAP technology as a case study, we will step through the low-power design techniques that have enabled the creation of a sensor consuming $<10 \mu \mathrm{W}$ with better than $1 \%$ full scale accuracy meeting typical automotive tire-pressure and temperature requirements. With the proposed multiplexed multi-slope procedure, a product designer has the ability to interface with multiple capacitive sensors and provide the readout on a single output line.
\end{abstract}

\section{Background:}

Electronic sensors are used in a wide variety of applications for measurement and control systems. Accuracy, reliability, and low costs are frequent expectations and driving factors for users. In past years, most user systems were built around commercially available sensors with analog type signal outputs using conveniently -- available power sources. Through the years, many user systems have migrated towards digital controls and microcontrollers to solve more complex problems and create new functionalities. A number of system applications have recently immerged requiring low-cost sensors that operate remotely with battery or battery-less power that must interface with low-power microcontrollers. Cost, compatibility with a differing power sources, low-power consumption, smaller size, and interfacing are important design considerations for modern sensors.

\section{General:}

Today, there are many different types of electronic sensors. Typically, a sensor signal needs offset, gain (span), linearity and temperature corrections or compensations. This is done by analog signal processors or by converting the analog sensor signal into the digital domain followed by a digital signal processor. A microcontroller is an example of such a digital signal processor.

Microcontrollers are manufactured in very high volume using advanced integrated circuit (IC) processes, resulting in optimal cost efficiencies. The manufacturing of sensors, however, can seldom be done in the same integrated circuit process because sensor manufacturing requires dedicated extra processing steps or even a completely different process. Therefore sensors, MEMS elements included, are commonly manufactured in special processes - so the signal processor (microcontroller) and the sensor cannot be realized on the same chip (IC). This partitions the design and a signal transfer protocol is required between the sensor and the signal processor.

Sensor signals can be very weak and usually need "- signal conditioning -" before the signals can be transferred to the signal processor. Examples of sensor signal conditioning circuits are amplifiers, integrators and Analog-to-Digital Converters (ADC). To build the most cost-effective measuring system, the strategy has been to minimize the amount of electronic circuitry on the sensor and rely on the signal processor because the processor typically has greater computation power and can be manufactured more cost-effectively.

Batteries are frequently used as the power supply of electronic sensors. Extremely low power consumption is important in applications where it is difficult to replace batteries. In battery applications, the sensor signal should be insensitive to battery voltage fluctuations that may occur with battery age and use. Another category of sensor applications uses an electro-magnetic field to power the sensor. The field injects charge into a supply-voltage capacitor via a coil and a rectifier. This type of application has even more extreme demands for low-power consumption and insensitivity to supply-voltage variation.

\section{Case Study Discussion:}

An integrated capacitive MEMS structure with on-chip conditioning circuitry (SCAP) was selected for the Kavlico sCAP ultra-low power sensing products because it provides significant advantages such as: 1) the MEMS sensor can be manufactured inexpensively with common CMOS processes; 2 ) the capacitive sense element can be excited and detected with significantly less power than resistive type devices; 3 ) the size of the capacitive sensing element can be made small because the signal to noise ratio of the sense element is large and by placing the conditioning 
circuitry on-chip, the parasitic "stray" capacitances of the circuit are managed; and 4) the conditioning circuitry creates a digital output signal that can be directly measured by a standard counter input of a microcontroller.

The PWM converter is a key element of a capacitive MEMS sensor that converts capacitances to electrical pulse durations. PWM converter circuits such as the Dual-Slope ADC and the Delta-Sigma ADC are commonly used in conditioning circuits. Both of these function but have limitations when used with a MEMS sensor. The Kavlico MultiSlope modulator was specially developed for the capacitive MEMS sensor. It is suited for the smaller capacitances of the MEMS elements and it improves on both the Dual-Slope and Delta-Sigma modulators.

The Kavlico sCAP MEMS element sense capacitors are in the order of 1 picofarad (pF). The minimum feasible integration current for use in circuitry is typically in the order of 10 to $100 \mathrm{nA}$. This can produce a detected signal pulse-width of only 10 to $100 \mu$ s with a $1 \mathrm{pF}$ element. These pulse-widths are not sufficient for adequate resolutions with a Dual-Slope converter circuits. A Multi-Slope modulator does not have the same restrictions. It supplies new data to the microcontroller with every completed A1ref and A1sens-phase (described below) and can be accumulated by the microcontroller. The software in the microcontroller can respond to fast signal changes or it can calculate a moving average.

A drawback of both the Multi-Slope and Dual-Slope conversions is that the integrator has a triangle shape output signal, which requires a relatively high bandwidth for the downstream operational amplifier. In case of a 2-stage operational amplifier with Miller compensation, like that chosen for the SCAP sensor, an effective solution to improve power efficiency is to not only switch the current at the input of the integrator, but to switch a small current to the Miller capacitor of the operational amplifier as well.

Figure 1 shows the Multiplexed Multi-Slope Conversion (MMSC) protocol for sensor signals: A1, A2, A3 and A4. The conversion starts when the supply voltage is applied to the circuit - or if an optional Sensor Select signal is applied. This starts the initialization phase of the electronic circuits, including the setting of the integrator output at its starting point. During the initialization phase, the output is set high. When the initialization is completed, the output is set low and the A1 Reference phase (A1ref) is started. The integrator output makes one up-slope and one down-slope during the A1ref-phase and one up-slope and one down-slope in the A1sens-phase. Both the reference phase and the sensing phase make one up-slope and one down-slope. This is an improvement compared with conventional Dual-Slope, eliminating the need for circuit behavior while integrating up closely to matches the behavior while integrating down. This improves the correlated double sampling effect. Optimal correlated double sampling cancels out non-ideal effects like circuit offset, clock feed-through of switches, low frequency noise, long-term drift of the electronic circuits and more.

During the first A1ref-phase and A1sens-phase, the integration coefficient selected has a low sensitivity to the second sensor signal A2. The ratio between the time of the A1ref-phase and that of the A1sens-phase is the unit of measure of $A 1$. The sum of the time of the first A1ref-phase and the first A1sens-phase is the first A2ref-phase. The conversion process of $A 1$ ref and $A 1$ sens is repeated by using an integration coefficient, which is affected by a relative high sensitivity to sensor signal $A 2$. This has no effect on the ratio between the second $A 1$ ref and A1sens, while the sum of the time of the second $A 1$ ref and $A 1$ sens phase is the first $A 2$ sens-phase. The ratio between the time of the A2ref-phase and that of the A2sens-phase is the unit of measure of A2. Next, the third A1ref-phase is started, which is part of the second A2ref-phase. And so on.

After giving a Sensor Select command, the microcontroller has to wait until the sensor output goes low, which is the end of the initialization phase. Then, the microcontroller starts a counter. At every clock, where the sensor output is high, the counter is increased by 1 . If the counter is at 400 after 1000 counts, it means that the A1ref-phase divided by the A1sens-phase is 600/400. As more counts that are taken, the accuracy increases. Also, there is no need to synchronize the clock of the microcontroller with the PWM-signal. The asynchronous clocking creates a negligible error if enough counts are taken. This is different than Delta-Sigma modulators, where synchronous clocking is required between the modulator and the signal processor. A Delta Sigma modulator requires an extra control line between the sensor and the signal processor for the synchronization.

To find the A2 data the microcontroller needs to start a second counter. This counter is increased by 1 during the even A1ref and A1sens phases: e.g. the $2^{\text {nd }}, 4^{\text {th }}, 6^{\text {th }}$, etc. - A1ref and A1sens phases -. If this counter has a value of 750 after 1000 counts, then the A2ref divided by the A2sens is $250 / 750$.

The Multiplexed Multi-Slope Conversion (MMSC) process, selected for the sCAP sensor, Figure 3, was designed for digital communication between the sensor and the digital signal processor. However, like Delta-Sigma converters, it can also be used as an analog interface between the sensor and an analog signal processor with the addition of a low-pass filter. The low-pass filter converts the PWM output to an analog output. The output value is equal to the duty cycle of the PWM-signal multiplied by the supply voltage. The low-pass filter can be as simple as an RC-filter. The drawback of this conversion to analog is that fast pressure changes cannot be detected anymore and the RCfilter consumes power (on average Vsupply/(2R)) so R must be very high in ultra-low-power applications. The input impedance of the analog signal processor must be at least two orders of magnitude higher than this R-value. 
Both pressure and temperature can be sensed with the sCAP structure as shown in Figure 2. Cp is an integrated pressure dependent capacitor (MEMS element) and $\mathrm{Cr}$ is the reference capacitor of the sensor. The circuit around the operational amplifier OP1 is an integrator. In this realization of the MMSC modulator, $\mathrm{Cp}$ and $\mathrm{Cr}$ are alternately used as the integrator capacitor. During Pressure-phases switch Scp is closed and switch Scr is open; during Prefphases, switch Scr is closed and Scp is open. A bias circuit ${ }^{1}$ injects a constant current in the integrator. To ramp up the integrator output, the bias circuit sinks current and sources current to ramp it down. The comparator circuit detects if the integrator output exceeds $\mathrm{VrefH}$ or VrefL, where after the control block takes care of the appropriate switch sequences and output value of the MMSC modulator. During Pref phases, the output is set Low and during P-phases the output is set High.

The bias circuit generates two different currents: Iref and I_T. Iref has a relative low TC and I_T has a relative high TC. Iref is used during the Tref-phases and I_T is used during the T-phases. In any IC process, resistive layers with different TC's are available and can be used to define Iref and I_T. For example, the poly-silicon layer can be used as the low TC resistor layer and the PWELL or NWELL layer can be used as the high TC resistor layer. The MMSC modulator starts with Iref as the integrator current during the Pref-phase as well as the P-phase and then it uses the I_T current for the next Pref-phase and P-phase, then Iref, etc. So every odd Pref and P phase of the modulator generates Tref-data and during every even Pref and P phase the modulator generates T-data.

To activate the circuit, a supply voltage is applied, or the optional Chip Select (CS) signal is set high to activate the initialization process. Switches Sini, Scpini and Scrini are closed, which presets the appropriate voltages at both terminals of $\mathrm{Cp}$ and $\mathrm{Cr}$. The initialization block is a timer circuit which starts the pressure and temperature sensing process if all bias voltages and bias currents are settled after start-up. During the initialization process, the output is set high. To minimize start-up effects, it is worthwhile to run one or more pressure and temperature cycles before the output of the MMSC modulator is released.

Note that during a Pref-phase and a P-phase, the exact same integration sourcing current, the exact same integration sinking current, the exact same operational amplifier and the exact same comparator are used; moreover a good matching between $\mathrm{Sp}$ and $\mathrm{Sr}$ can be easily accomplished in the integrated circuit process. This produces a nearly perfect correlated double sampling during the Pref-phase and the P-phase, eliminating the offset, long-term offset drift, and the low frequency noise of the active circuit elements. High frequency noise will cause a jitter on the output of the MMSC modulator, but this effect is cancelled out by calculating the (moving) average of the PWM output.

A digital signal processor controls the MMSC modulator. When it starts, the MMSC modulator it has to wait until the first falling edge of the MMSC output. Then, it starts the counter, which counts the:

1) total number of clocks of the internal clock of the processor while the MMSC modulator is activated,

2) number of clocks that modulator output is high, and

3) number of clocks that the modulator is using I_T as the integrator current. This is the total time of the even Pref and P-phases.

The software stored in the digital signal processor defines the algorithm to convert the above data into actual pressure and temperature. In a simple form, the pressure and temperature are equal to:

$$
\begin{array}{ll}
\mathrm{P} & =\mathrm{psp}{ }^{*}(\text { Psens }- \text { poff * Pref }) / \text { Psens } \\
\mathrm{T} & =\mathrm{tsp}{ }^{*}\left(\text { Tsens }- \text { toff }^{*} \text { Tref }\right)
\end{array}
$$

Where psp is the pressure span (gain) calibration coefficient, poff is the pressure offset calibration coefficient, tsp is the temperature span calibration coefficient, and toff is the temperature offset calibration coefficient. Typically, these calibration coefficients will be stored in the memory of the signal processor.

\footnotetext{
${ }^{1}$ The symbol of the bias circuit is symbolic only. Current sources cannot be in series with an open switch. The current source needs to be turned off or switched to a low impedance node. For the sake of simplicity, this is not shown in the symbol.
} 


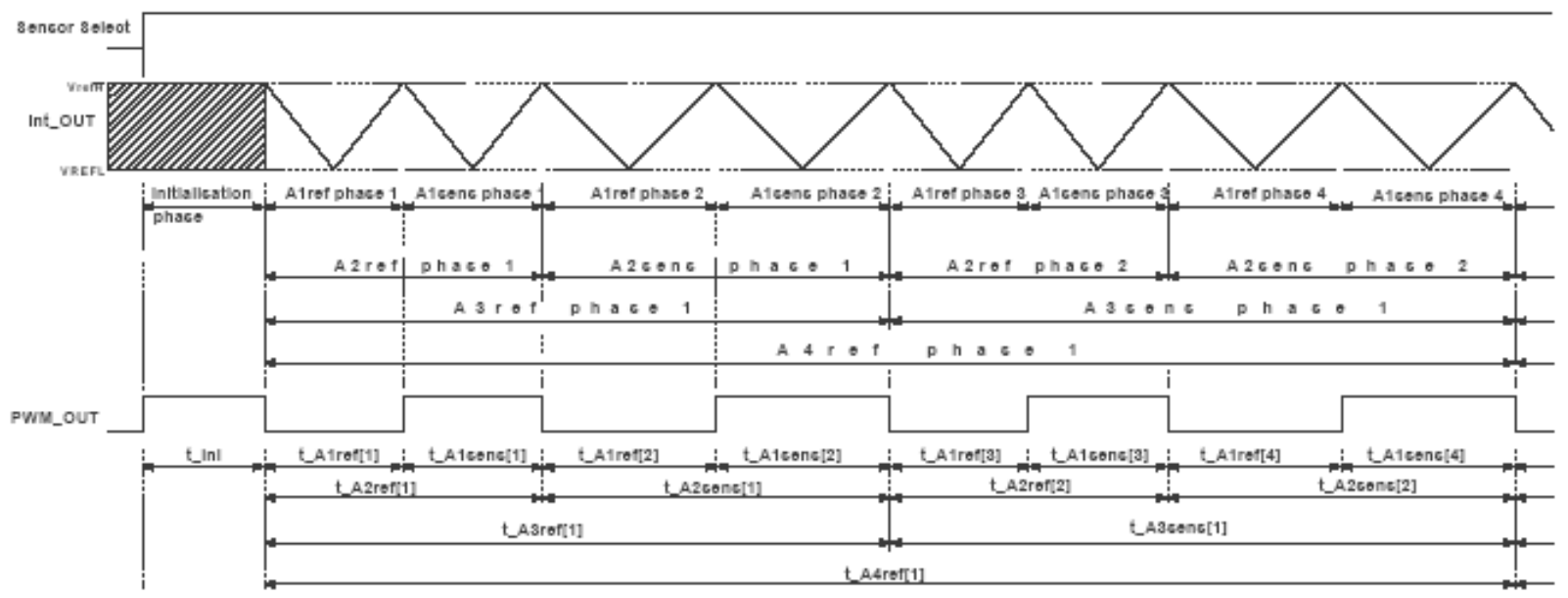

Figure 1 - MMSC Protocol

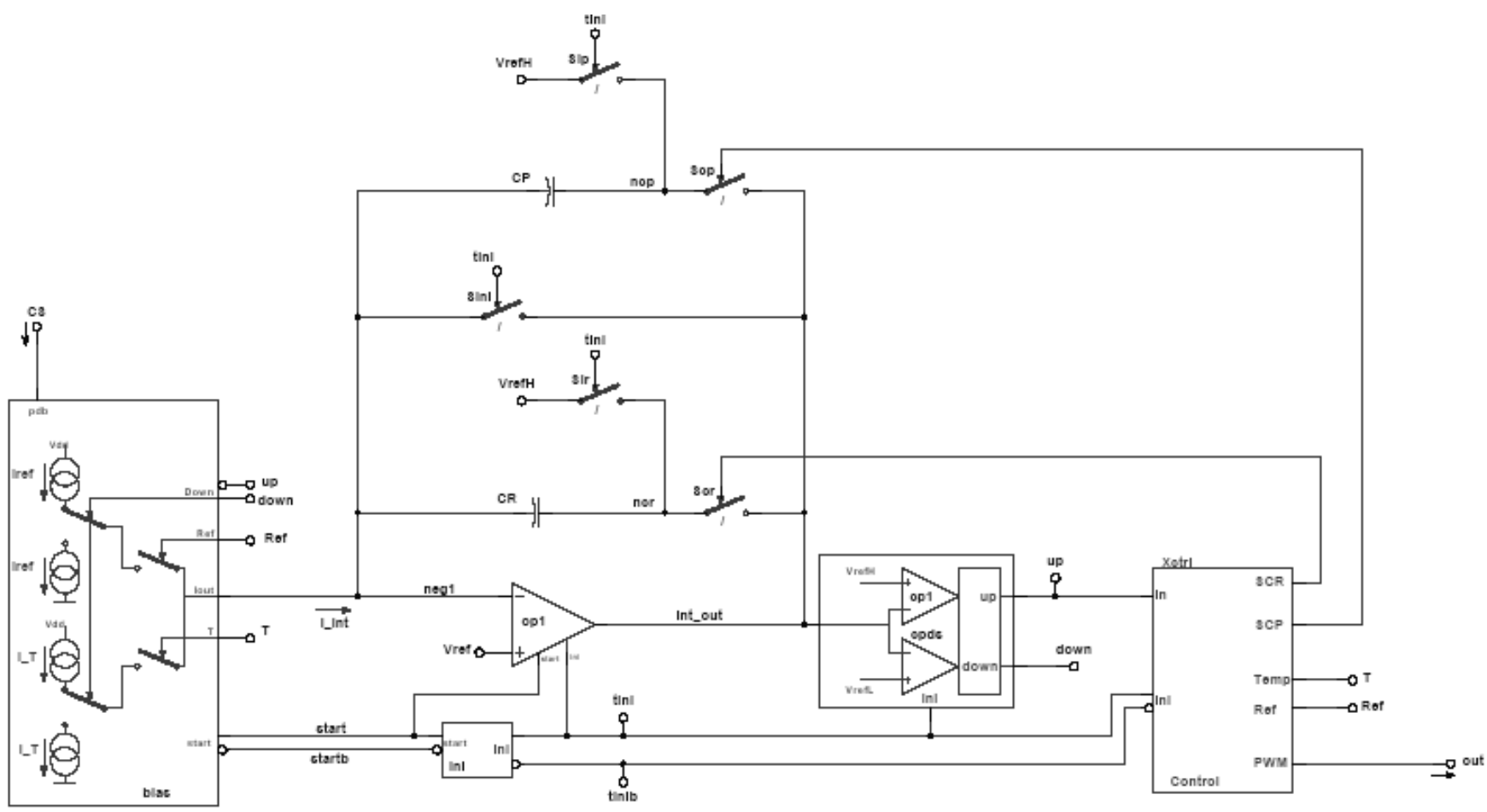

Figure 2 - Pressure and Temperature MMSC Modulator Circuit (sCAP Sensor) 

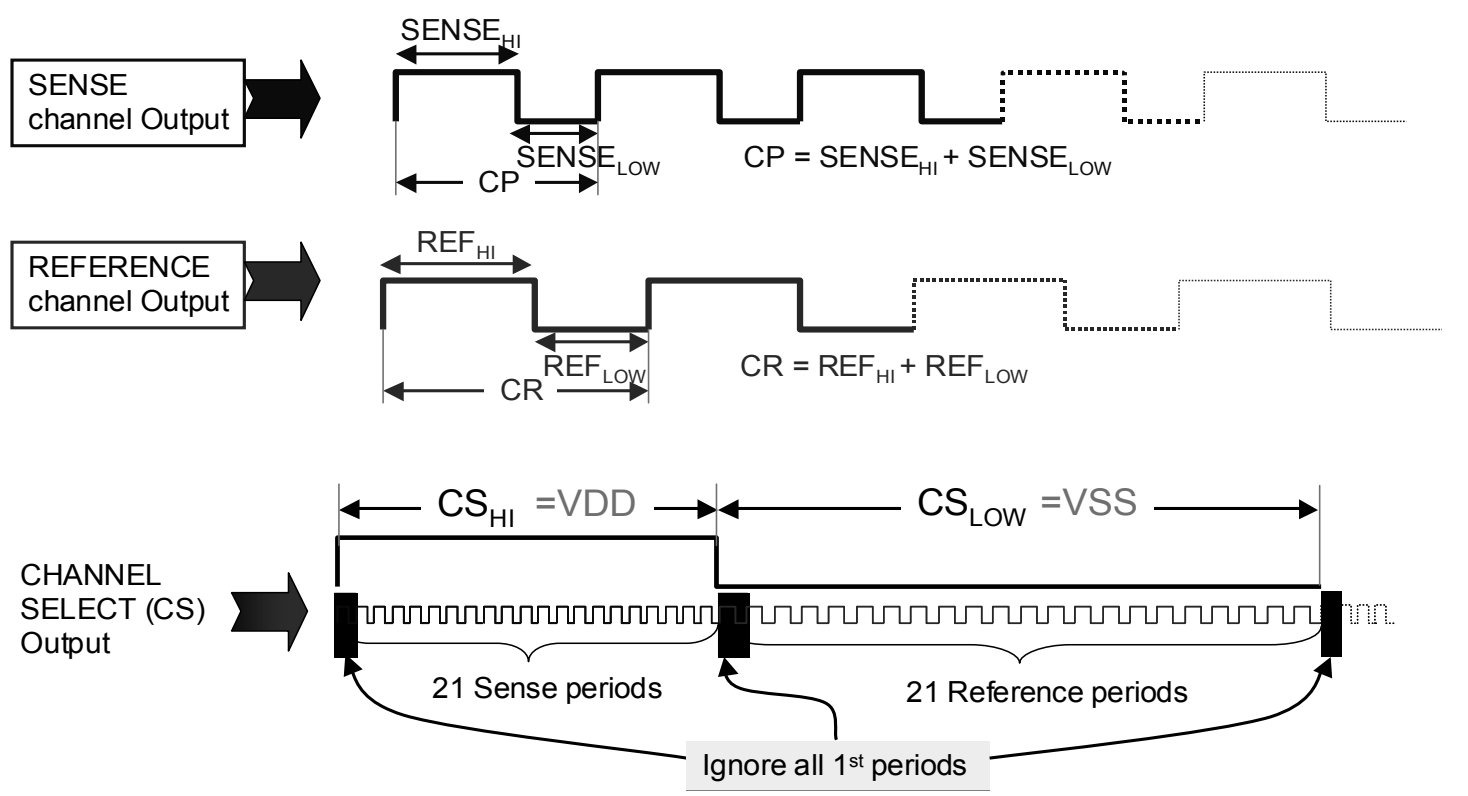

Setting the CS pin to VDD triggers SENSE output Setting the CS pin to VSS triggers REFERENCE output

Figure 3 - Pressure and Temperature Measurement Protocol (sCAP Sensor)

SCAP TRANSFER FUNCTION EQUATIONS FOR PRESSURE AND TEMPERATURE OUTPUT:

1. $\mathrm{C}_{\mathrm{SPT}}=\mathrm{P} \_$Span $\cdot\left[1+\mathrm{TC} \_\mathrm{Span} \cdot(\mathrm{TX}-25)\right]$

2. $\mathrm{C}_{\mathrm{OFFT}}=\mathrm{P} \_$Offset $\cdot\left[1+\mathrm{TC} \_\right.$Offset $\left.\cdot(\mathrm{TX}-25)\right]$

3. $\mathrm{P}=\frac{\mathrm{C}_{\mathrm{SPT}} \cdot\left[\mathrm{AvgCP}-\left(\mathrm{C}_{\mathrm{OFFT}} \cdot \mathrm{AvgCR}\right)\right]}{\mathrm{AvgCP}-\left(\mathrm{P}_{-} \text {Linearity } \cdot \mathrm{AvgCR}\right)}$

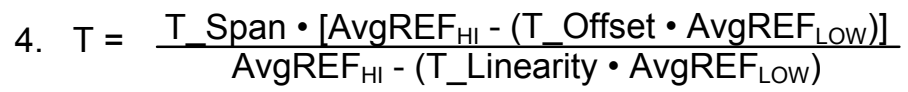

Where:

$\mathrm{P}=\quad$ Pressure Data Signal (Calculated)

$\mathrm{T}=\quad$ Temperature Data Signal (Calculated)

$\mathrm{Tx}=\quad$ Test or Measurement temperature $\left({ }^{\circ} \mathrm{C}\right)$ or calculated

$P_{-}$Linearity $=\quad$ Pressure Linearity (Factory supplied coefficient)

P_Offset $=\quad$ Pressure Offset (Factory supplied coefficient $)$

P_Span $=\quad$ Pressure span (Factory supplied coefficient)

$\bar{T} \bar{C}$ Offset $=\quad$ Temperature Compensated Offset (Factory supplied coefficient)

TC_Span $=\quad$ Temperature Compensated span (Factory supplied coefficient)

T_Linearity $=\quad$ Temperature Linearity (Factory supplied coefficient)

T_Offset $=\quad$ Temperature Offset (Factory supplied coefficient)

T_Span $=\quad$ Temperature Span (Factory supplied coefficient)

AvgCP $\quad \quad$ Average of $\geq 20 \mathrm{CP}$ periods (Calculated)

AvgCR $=\quad$ Average of $\geq 20 \mathrm{CR}$ periods (Calculated)

AvgREF $_{\mathrm{HI}}=\quad$ Average of $\geq 20 \mathrm{REF}_{\mathrm{HI}}$ periods (Calculated)

AvgREF$_{\text {Low }}=\quad$ Average of $\geq 20 \mathrm{REF}_{\text {Low }}$ periods (Calculated)

$\mathrm{C}_{\mathrm{OFFT}}=\quad$ Temperature Compensation Offset Pressure Coefficient (Calculated)

$\mathrm{C}_{\mathrm{SPT}}=\quad$ Temperature Compensation Span Pressure Coefficient (Calculated) 


\section{SCAP SENSOR CALIBRATION/ COMPENSATION STRATEGY:}

The pressure output data is encoded in the period of the waveform. The temperature output data is encoded in the pulse widths of the waveform.

To extract the pressure output signal data, the following procedure is suggested:

1. After a minimum of $500 \mu S$ following power turn-on. Set the Channel Select (CS) input to the "VSS" level.

2. After a minimum delay of one output waveform period, begin measuring the duration of the periods (CR).

3. Accumulate and average a minimum of twenty periods.

4. Calculate the average period and store it as the pressure measurement (AvgCR).

5. Set the Channel Select (CS) input to a "VDD" level.

6. After a minimum delay of one waveform period, begin measuring the duration of the periods $(C P)$.

7. Accumulate and average a minimum of twenty periods.

8. Calculate the average period and store it as the pressure reference ( $\mathrm{AvgCP})$.

To extract the temperature output signal data, the following procedure is suggested:

1. After a minimum of $500 \mu S$ following power turn-on. Set the Channel Select (CS) input to the "VSS" level.

2. After a delay of one waveform output period, begin measuring the duration of the pulses $\left(R E F_{H I}, R_{E} F_{L O W}\right)$.

3. Measure the duration of the "on-time" of the waveform $\left(\mathrm{REF}_{\mathrm{HI}}\right)$.

4. Accumulate and average a minimum of twenty "on" $\left(\mathrm{REF}_{\mathrm{HI}}\right)$ pulse durations.

5. Calculate the average and store as the temperature sensed measurement $\left(A v g R E F_{H I}\right)$.

6. Measure the "off-time" (REFLow) of the waveform.

7. Accumulate and average a minimum of twenty "off" pulse durations (REFLOW).

8. Calculate the average and store as temperature reference measurement (AvgREFLow).

Performance Specifications for Case Study:

\begin{tabular}{|c|c|c|c|c|}
\hline Specification & Minimum & Typical & Maximum & Units \\
\hline Supply Voltage & 1.8 & 3 & 10 & $\mathrm{~V}$ \\
\hline Power Consumption & 10 & 75 & 200 & $\mu \mathrm{W}$ \\
\hline Operating Temperature & -50 & 25 & 150 & ${ }^{\circ} \mathrm{C}$ \\
\hline Response Time & 50 & 250 & 600 & $\mathrm{MS}$ \\
\hline Long Term Drift & & & 0.5 & $\%$ \\
\hline Pressure Operation & 15 & 100 & 10,000 & $\mathrm{PSIA}$ \\
\hline
\end{tabular}
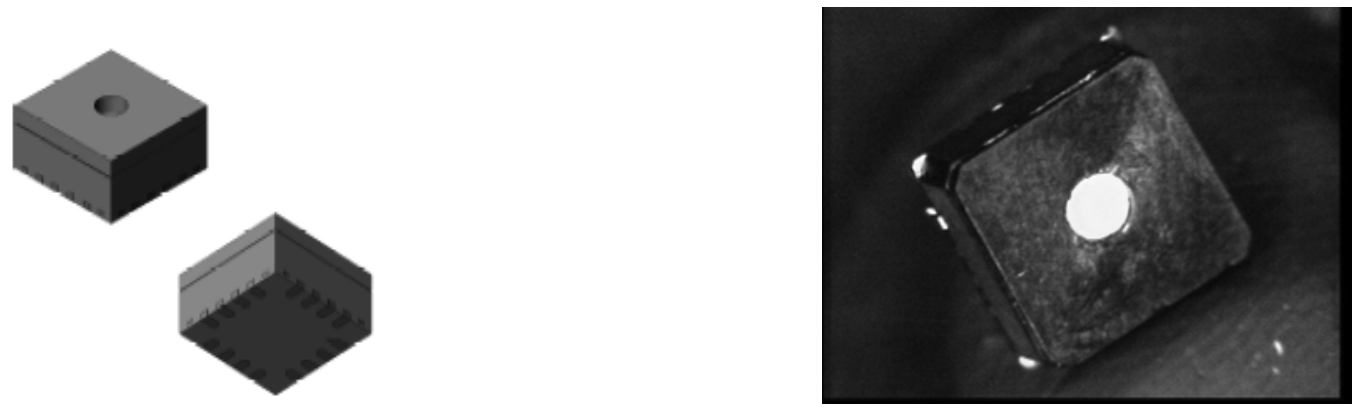